\title{
LONG-TERM VARIABILITY IN THE QUIESCENT X-RAY TRANSIENT V404 CYG
}

\author{
C. Zurita, ${ }^{1}$ J. Casares,${ }^{2}$ P. A. Charles ${ }^{3}$ R. I. Hynes,${ }^{4}$ E. P. Pavlenko, ${ }^{5}$ and T. Shahbaz ${ }^{2}$
}

\begin{abstract}
We present the results of optical and infrared photometry of the quiescent X-ray transient V404 Cyg during the period 1992-2003.
\end{abstract}

It is largely known that the Soft X-ray Transient (SXT) V404 Cyg exhibits short-term (sub-orbital) variations during quiescence (see eg. Pavlenko et al. 1996). It seems that fast variability during quiescence is not a peculiarity of V404 Cyg but a fingerprint of quiescent SXTs, irrespective of the nature of the compact object (Zurita, Casares \& Shahbaz, 2003). Although these variations seem to originate in the accretion disc, the underlaying physical mechanism is not yet understood. Since V404 Cyg is the brightest SXT, and hence accessible with 1-m type telescopes, we embarked on a study of the fast variability over the period 1992-2003.

We obtained $R$ band CCD photometry of V404 $\mathrm{Cyg}$ with various $1 \mathrm{~m}$-class telescopes during the years 1992, 1998, 1999, 2001, 2002 and 2003. V404 Cyg was obserred in 1998 simultaneously in the $R$ and $I$ band and also in $2001 R$-band, $J$ and $K_{s}$ simultaneous observations were performed.

The ellipsoidal modulations extracted from the most complete databases (years 1992, 1998 and 2001) show unequal maxima and minima with relative strength varying from year to year (see lower envelopes in Fig.1). These distortions could be explained assuming changes in the accretion disc geometry and brightness due suprhumps or spots on the surface of the secondary star. Fast optical (flarelike) variations superimposed on the secondary star's double-humped ellipsoidal modulation were detected every year with a mean amplitude of $\sim 0.07$ mags (dispersion above the lower envelope in Fig. 1). We found in many nights large single events of $\sim 2 \mathrm{~h}$ or more duration appearing together with faster and small amplitude variations. In order to characterize the flares we isolated them by subtracted the ellipsoidal modulation and we have not found significant changes in the activity during this decade

\footnotetext{
${ }^{1}$ CAAUL, Observatório Astronómio de Lisboa, Tapada da Ajuda 1349-018 Lisboa, Portugal (czurita@oal.ul.pt).

${ }^{2}$ Instituto de Astrofisica de Canarias, Spain.

${ }^{3}$ University of Southampton, UK.

${ }^{4}$ University of Texas at Austin, USA.

${ }^{5}$ Crimean Astrophysical Observatory, Ukraine.
}

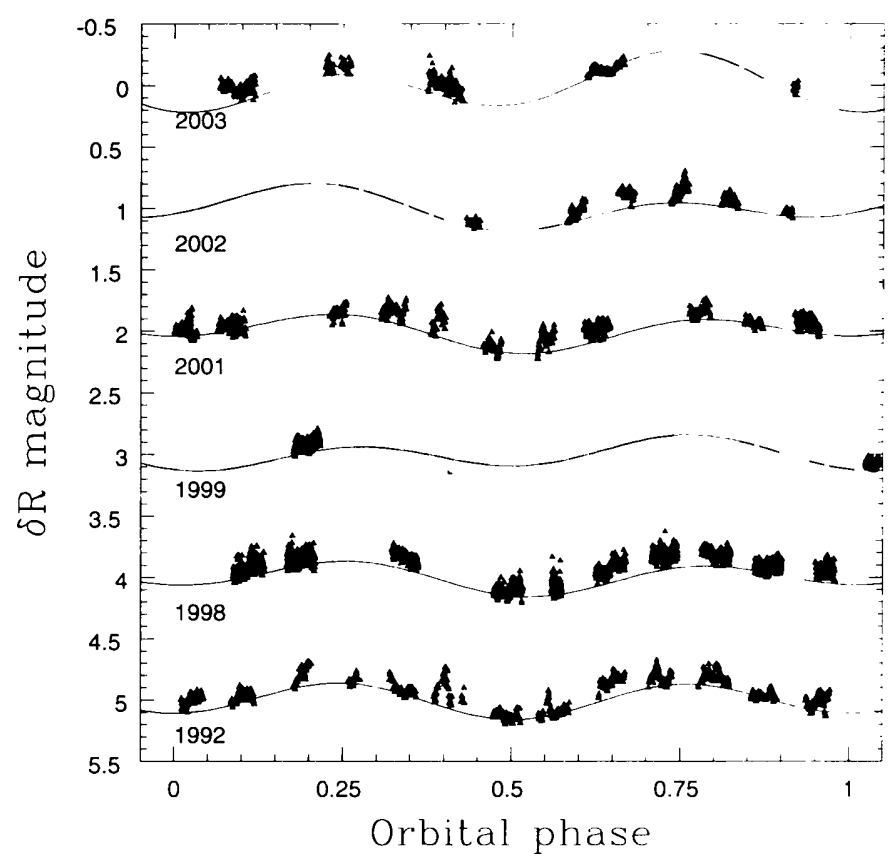

Fig. 1. The $R$ band ellipsoidal lightcurves of V404 Cyg for 1992-2003 and the lower envelopes. The orbital period of V404 Cyg is $6.5 \mathrm{~d}$ (Casares et al. 1992). Different magnitude offsets were aplied for clarity.

which indicates that this variability is probalbly not connected to the 1989 outburst. We have found periodicities in the 1998 and 2001 data near the $6 \mathrm{hr}$ quasi-periodicity observed in 1992, although we interpret it as consequence of the appearance of a flare event almost every night.

We have also found significant variability in the $I$ and near-IR band $\left(J\right.$ and $\left.K_{s}\right)$ and this is correlated. In particular a cross correlation analysis shows that both the $R$ and $I$ emission are simultaneous down to $40 \mathrm{~s}$. We also calculated the fluxes of the flares in all bands and obtained $F_{R} / F_{I} \sim 3.0, F_{R} / F_{J} \sim 1.8$ and $F_{J} / F_{K_{s}} \sim 3.0$. However, we could only obtain a rough estimation due the large uncertainties in the determination of the reddening.

\section{REFERENCES}

Casares J., Charles P.A., Naylor T., 1992, Natur, 355. 614

Pavienko E.P., Martin A.C., Cancures J., Charles P.A. Ketsaris N.A.. 1996, MNRAS, 2\&1, 1094

Zurita C. Casares J. \& Shahbaz T., 2003, ApJ, 52:. :36? 